PROCEEDINGS OF THE

AMERICAN MATHEMATICAL SOCIETY

Volume 125, Number 6, June 1997, Pages 1793-1799

S 0002-9939(97)03776-3

\title{
CONVEXITY AND HAAR NULL SETS
}

\author{
EVA MATOUŠKOVÁ
}

(Communicated by Dale Alspach)

\begin{abstract}
It is shown that for every closed, convex and nowhere dense subset $C$ of a superreflexive Banach space $X$ there exists a Radon probability measure $\mu$ on $X$ so that $\mu(C+x)=0$ for all $x \in X$. In particular, closed, convex, nowhere dense sets in separable superreflexive Banach spaces are Haar null. This is unlike the situation in separable nonreflexive Banach spaces, where there always exists a closed convex nowhere dense set which is not Haar null.
\end{abstract}

A Borel subset $A$ of a separable Banach space $X$ is called a Haar null set if there exists a probability measure $\mu$ on the $\sigma$-algebra of Borel subsets of $X$ so that $\mu(A+x)=0$ for all $x \in X$ (see [C] also for the following properties of Haar null sets). The family of all such sets is closed under translation and under countable unions; nonempty open sets do not belong to it. The Haar null sets agree with Lebesgue null Borel sets in finite dimensional spaces. This definition of null sets is rather weak, as every compact set in an infinite dimensional space is a Haar null set. In fact, in infinite dimensional superreflexive and nonreflexive Banach spaces even all weakly compact convex sets with empty interior are Haar null. For superreflexive spaces this follows from our result. If $K$ is a weakly compact and convex subset of a nonreflexive Banach space, then $\bigcup_{t>0} t(K-K) \neq X$ and there exists $x \in X$ so that the intersection of the line segment $[0, x]$ and any translate of $K$ contains at most one point. Consequently, if $\mu$ is Lebesgue measure on $[0, x]$, then $\mu(K+x)=0$ for each $x \in X$. In [MS] it is shown that a separable Banach space is nonreflexive if and only if there exists a closed convex subset $Q$ of $X$ with empty interior, which contains a translate of any compact subset of $X$. Such a set $Q$ is not Haar null because given any probability measure $\mu$ on $X$ there exists a compact set $K$ with $\mu(K)>0$ and, consequently, also a translate of $Q$ of positive measure. It follows that every separable nonreflexive Banach space contains a closed convex set with empty interior which is not Haar null. In this note we show that this is unlike the situation in superreflexive spaces, where for every closed convex set $C$ with empty interior there exists a Radon probability measure $\mu$ on $X$ so that $\mu(C+x)=0$ for all $x \in X$. We do not know if such a measure exists when $X$ is only reflexive or, equivalently, if the positive cone of every reflexive Banach space with a basis is Haar null.

Received by the editors February 22, 1995 and, in revised form, January 8, 1996 1991 Mathematics Subject Classification. Primary 46B10; Secondary 46B20.

Key words and phrases. Superreflexive Banach spaces, convexity, Haar null sets.

The author was partially supported by the grant GAČR 201/94/0069 and by a grant of the Austrian Ministry of Education.

(C)1997 American Mathematical Society 
There are several results dealing with the question of how often a convex continuous function on a Banach space is differentiable. Let us mention a few examples of the "almost everywhere" ones. In the finite dimensional spaces the theorem of Rademacher says that every locally Lipschitz (and, hence, every convex and continuous) function is almost everywhere Fréchet differentiable. In separable Banach spaces the substitute for this theorem is Aronszajn's theorem [A], which says that every locally Lipschitz function is Gâteaux differentiable outside of an Aronszajn null set. This does not hold for Fréchet differentiability even in reflexive spaces as the Hilbert cube $D=\left\{x \in \ell_{2}: 0 \leq x_{n} \leq 1 / n\right\}$ is not Aronszajn null (cf. [A]) and the distance function to $D$ (which is convex and continuous) is not Fréchet differentiable at any point of $D$. One may wonder if it would help to replace the Aronszajn null sets by some larger class of null sets, for example, the Haar null sets. This is not the case for functions which are just Lipschitz; from [PT] it follows that on every separable Banach space there exists a Lipschitz function for which the set of points where it is not Fréchet differentiable is not Haar null. In every separable nonreflexive space, there even exists a convex continuous function with this property. Indeed, the distance function to a closed convex subset $K$ of a Banach space is not Fréchet differentiable at any point of the boundary of $K$, so the distance function to a closed convex set which is not Haar null and has empty interior will do as an example. We do not know whether such an example exists in separable reflexive spaces. From our result, which answers negatively a conjecture in [BN], it follows that in separable superreflexive space one can not construct an example as above since the boundaries of convex sets are Haar null. For sets with nonempty interior this is true in any separable Banach space. Indeed, it is easy to see that if $K$ is a closed convex set with the origin in the interior then the supremum of the Minkowski functional of $K$ and the constant function equal to one is not Gâteaux differentiable at any point of the boundary of $K$. Consequently, the boundary is, by Aronszajn's theorem, Aronszajn null and hence also Haar null. If the interior of $K$ is empty, this trick does not always work. If $K$ is not Aronszajn null (for example, take again the Hilbert cube $D$ in $\ell_{2}$ as above) then every convex continuous function is Gâteaux differentiable also at some points of $K$. Finally, let us remark, that if instead of Fréchet smoothness we consider the stronger notion of Lipschitz smoothness then there exists a convex continuous function on $\ell_{2}$ such that the set of points where it is not smooth is not Haar null [MZ].

Let $X$ be a Banach space with a Schauder basis $\left\{x_{n}\right\}$. We say that the basis is normalized, if $\left\|x_{n}\right\|=1$ for all $n \in N$. By the positive cone of $X$ we mean the (necessarily closed and convex) set $Q=\left\{\sum_{i=1}^{\infty} \alpha_{i} x_{i} \in X: \alpha_{i} \geq 0\right.$ for $\left.i=1,2, \ldots\right\}$. The characteristic of the basis is the largest of all $\varepsilon \geq 0$ for which $\left\|\sum_{i=1}^{n} a_{i} x_{i}\right\| \geq$ $\varepsilon\left\|\sum_{i=1}^{k} a_{i} x_{i}\right\|$ if $n>k$ and $\left\{a_{i}\right\}$ is any sequence of numbers; it holds always that $0<\varepsilon \leq 1$. A Radon measure on a Banach space $X$ is a measure on the $\sigma$-algebra of Borel subsets of $X$, which is inner regular with respect to the compact sets. We say that a convex subset $C$ of a Banach space $X$ is spanning if it contains a line segment in every direction, that is,

$$
\bigcup_{t>0} t(C-C)=X
$$

In [MS] it is shown that if $Q$ is a closed, convex, bounded, spanning and nowhere dense subset of a Banach space $X$, and $T: X \rightarrow Y$ is a surjective, continuous and 
linear operator with finite dimensional kernel, then $T(Q)$ is also a closed, convex, bounded and spanning subset of $Y$ with empty interior.

Lemma 1. Let $X$ be a weakly compactly generated Banach space, and $C \subset X a$ closed, convex, bounded and spanning set with empty interior. Then there exists an infinite dimensional quotient space $Y$ of $X$ with a Schauder basis and $v \in X$ so that the image of $C+v$ under the quotient mapping is contained in the positive cone of $Y$.

Proof. If $C$ is a closed, convex and nowhere dense subset of a weakly compactly generated Banach space $X$, then there exists a projection $P$ onto a separable subsace $Z$ of $X$ such that the interior of $\overline{P(C)}$ in $Z$ is empty (see Lemma 3). Therefore, we may suppose that $X$ is separable. Let us recall the following elementary fact: if $K$ is a closed, convex and nowhere dense subset of a Banach space then for every $\varepsilon>0$ there exists a norm one element $x^{*}$ of the dual so that $\left\langle x^{*}, x\right\rangle>-\varepsilon$ for all $x \in K$. Choose a sequence $\left\{x_{n}\right\}$ that is dense in the unit sphere of $X$. For $n \geq 1$ construct, by induction, a sequence $\left\{x_{n}^{*}\right\}$ in the unit sphere of $X^{*}$ and a sequence $\left\{v_{n}\right\}$ in $X$ as follows. Set $k_{0}=1$. Put $X_{n}=\operatorname{span}\left\{x_{1}, \ldots, x_{k_{n-1}}\right\}$ and choose a closed subspace $Z_{n}$ of $X$ so that $X_{n} \oplus Z_{n}=X$. Let $P_{n}: X \rightarrow Z_{n}$ be the projection with kernel $X_{n}$. Then $P_{n}^{*}$ is an isomorphism between $Z_{n}^{*}$ and the annihilator $X_{n}^{\perp}$ of $X_{n}$ in $X^{*}$; choose $c_{n}>0$ so that $c_{n}\left\|z^{*}\right\| \leq\left\|P_{n}^{*}\left(z^{*}\right)\right\|$ for each $z^{*} \in Z_{n}^{*}$. Let $C_{n}$ be the projection of $C$ in $Z_{n}$. Since $C$ is closed, convex, bounded, nowhere dense and spanning, $C_{n}$ is a closed convex set with empty interior by the result from [MS] mentioned above. Consequently, there exists $z_{n}^{*} \in Z_{n}^{*}$ so that

$$
\left\|z_{n}^{*}\right\|=1 \quad \text { and } \quad\left\langle z_{n}^{*}, z\right\rangle \geq-c_{n} / 4^{n+1}
$$

for each $z \in C_{n}$. Define $x_{n}^{*}=P_{n}^{*}\left(z_{n}^{*}\right) /\left\|P_{n}^{*}\left(z_{n}^{*}\right)\right\|$. Then $x_{n}^{*} \in X_{n}^{\perp},\left\|x_{n}^{*}\right\|=1$, and for each $x \in C$ we have that

$$
\begin{aligned}
\left\langle x_{n}^{*}, x\right\rangle & =\left\langle P_{n}^{*}\left(z_{n}^{*}\right), x\right\rangle /\left\|P_{n}^{*}\left(z_{n}^{*}\right)\right\|=\left\langle z_{n}^{*}, P_{n}(x)\right\rangle /\left\|P_{n}^{*}\left(z_{n}^{*}\right)\right\| \\
& \geq \frac{-c_{n}}{4^{n+1}\left\|P_{n}^{*}\left(z_{n}^{*}\right)\right\|} \geq \frac{-1}{4^{n+1}}
\end{aligned}
$$

since $P_{n}(x) \in C_{n}$. Now choose an integer $k_{n} \geq \max \left\{k_{n-1}, n+1\right\}$ and real numbers $\alpha_{i}, i=1, \ldots, k_{n}$, so that for $v_{n}=\sum_{i=1}^{k_{n}} \alpha_{i} x_{i}$, it holds that $\left\|v_{n}\right\| \leq 3 / 2$ and $\left\langle x_{n}^{*}, v_{n}\right\rangle \geq 1$. Since the spaces $X_{n}$ are increasing, their union is dense in $X$, and each $x_{n}^{*}$ annihilates $X_{n}$, it follows that the sequence $\left\{x_{n}^{*}\right\}$ converges to zero in the weak ${ }^{*}$ topology. Put $v=\sum_{i=1}^{\infty} 4^{-i} v_{i}$. Then $\left\langle x_{n}^{*}, x+v\right\rangle \geq 0$ for each $x \in C$ and each $n \in N$. Indeed, if $x \in C$, then

$$
\begin{aligned}
\left\langle x_{n}^{*}, x+v\right\rangle & =\left\langle x_{n}^{*}, x\right\rangle+\left\langle x_{n}^{*}, \sum_{i=1}^{n-1} 4^{-i} v_{i}\right\rangle+\left\langle x_{n}^{*}, 4^{-n} v_{n}\right\rangle+\left\langle x_{n}^{*}, \sum_{i=n+1}^{\infty} 4^{-i} v_{i}\right\rangle \\
& \geq-4^{-(n+1)}+0+4^{-n}-4^{-n} \sum_{i=1}^{\infty} 4^{-i}\left\|v_{n+i}\right\| \geq 0
\end{aligned}
$$

where the last inequality follows from the fact that $\left\|v_{i}\right\| \leq 3 / 2$. Since $X$ is separable and $x_{n}^{*}$ is a weak*-null sequence in the sphere of $X^{*}$, it has a subsequence which is weak* basic (cf. [LT], pg. 11). Without loss of generality, suppose that $\left\{x_{n}^{*}\right\}$ is already such a sequence. Let $\left\{x_{n}^{*}\right\}_{\perp}$ be the annihilator of $\left\{x_{n}^{*}\right\}$ in $X$. Then (again, see [LT] $)$, the quotient space $Y=X /\left\{x_{n}^{*}\right\}_{\perp}$ has a basis $\left\{y_{n}\right\}$ and $x_{n}^{*}=T^{*}\left(y_{n}^{*}\right)$, 
where $T: X \rightarrow Y$ is the quotient map and $\left\{y_{n}^{*}\right\}$ are the coeficient functionals of $\left\{y_{n}\right\}$. Then,

$$
\left\langle y_{n}^{*}, T(x+v)\right\rangle=\left\langle T^{*}\left(y_{n}^{*}\right), x+v\right\rangle=\left\langle x_{n}^{*}, x+v\right\rangle \geq 0
$$

for all $x \in C$ and $n \in N$. Consequently, the projection of $C$ is contained in the positive cone of $Y$.

The construction of the measure on $\ell_{1}$ in the proof of the following lemma is a modification of the proof in $[\mathrm{BN}]$ (p. 54), where it is shown that for $p \geq 1$ the positive cone of $\ell_{p}$ is Haar null.

Lemma 2. Let $X$ be a Banach space with a normalized Schauder basis $\left\{x_{n}\right\}$, and suppose there exist $p \geq 1$ and $K>0$ so that

$$
\left(\sum_{i=1}^{n}\left|\beta_{i}\right|^{p}\right)^{\frac{1}{p}} \leq K\left\|\sum_{i=1}^{n} \beta_{i} x_{i}\right\|
$$

for all real numbers $\beta_{i}, i=1, \ldots, n$ and $n \in N$. Then the positive cone $Q$ of $X$ is Haar null.

Proof. Firstly, let us construct a probability measure $\mu$ on the positive cone of $\ell_{1}$ such that whenever $\left(\beta_{n}\right)$ is a sequence in $\ell_{p}$ then

$$
\mu\left\{\left(\alpha_{n}\right) \in \ell_{1}: \alpha_{n} \leq \beta_{n}\right\}=0 .
$$

To this end, choose a point $\left(a_{n}\right)$ in $\ell_{p+1} \backslash \ell_{p}$ with each $a_{n}>0$; for example put $a_{n}=n^{-1 / p}$. Fix independent random variables $f_{n}:[0,1] \rightarrow[0,1]$ such that

$$
\begin{aligned}
& \lambda\left\{t \in[0,1]: f_{n}(t)=0\right\}=1-a_{n}^{p} \quad \text { and } \\
& \lambda\left\{t \in[0,1]: f_{n}(t)=a_{n}\right\}=a_{n}^{p},
\end{aligned}
$$

where $\lambda$ denotes Lebesgue measure. Let $F(t)=\left(f_{n}(t)\right)$ be the mapping from $[0,1]$ to $R^{\infty}$, the countable product of the reals with the product topology. Then $F$ is Borel measurable because it is coordinatewise measurable, and we can define a Borel probability measure $\nu_{o}$ on $R^{\infty}$ as follows:

$$
\nu_{o}(A)=\lambda\{t \in[0,1]: F(t) \in A\}
$$

for every Borel subset $A$ of $R^{\infty}$. Let $\nu$ be the completion of $\nu_{o}$. Consider the natural imbedding $g\left(\left(\alpha_{n}\right)\right)=\left(\alpha_{n}\right)$ of $\ell_{1}$ into $R^{\infty}$. Since $\ell_{1}$ is a complete metric space and $g$ is continuous, $g$ carries Borel subsets of $\ell_{1}$ to analytic subsets of $R^{\infty}$, and the latter, by a theorem of Szpilrajn-Marczewski, are $\nu$-measurable since $\nu$ is complete. (Or, more easily, observe that closed balls in $\ell_{1}$ are closed subsets of $R^{\infty}$; namely, $\bar{B}_{\ell_{1}}(0, r)=\bigcap_{n \in N}\left\{\left(x_{i}\right) \in R^{\infty}: \sum_{i=1}^{n}\left|x_{i}\right| \leq r\right\}$. Therefore, Borel subsets of $\ell_{1}$ are Borel also in $R^{\infty}$, since $\ell_{1}$ is separable.) Hence, if we define $\mu(B)=\nu(g(B)$ ) for Borel subsets of $\ell_{1}$, then $\mu$ is a Borel measure on $\ell_{1}$. To show that $\mu$ is a probability measure on $\ell_{1}$ it is enough to check that $\left(f_{n}(t)\right) \in \ell_{1}$ for almost all $t \in[0,1]$. This follows because we have

$$
\int_{0}^{1} \sum_{n=1}^{\infty}\left|f_{n}(t)\right| d t=\sum_{n=1}^{\infty} \int_{0}^{1}\left|f_{n}(t)\right| d t=\sum_{n=1}^{\infty} a_{n}^{p+1}<\infty
$$

and this proves that $\sum\left|f_{n}\right|<\infty$ almost everywhere. Hence $\mu\left(\ell_{1}\right)=1$. Fix $\left(\beta_{n}\right) \in$ $\ell_{p}$. If $\beta_{n}<0$ for some $n$, then, clearly, $\mu\left\{\left(\alpha_{n}\right) \in \ell_{1}: \alpha_{n} \leq \beta_{n}\right\}=0$; otherwise, 
define

$$
\begin{aligned}
\theta=\mu\left\{\left(\alpha_{n}\right) \in \ell_{1}: \alpha_{n} \leq \beta_{n}\right\} & =\lambda\left\{t \in[0,1]: f_{n}(t) \leq \beta_{n} \text { for all } n\right\} \\
& =\prod_{n=1}^{\infty} \lambda\left\{t \in[0,1]: f_{n}(t) \leq \beta_{n}\right\} \\
& =\prod_{\beta_{n}<a_{n}}\left(1-a_{n}^{p}\right) .
\end{aligned}
$$

Clearly, $\theta=0$ if $\eta=\sum_{\beta_{n}<a_{n}} a_{n}^{p}=\infty$. Since $\left(a_{n}\right) \notin \ell_{p}$, if $\eta<\infty$ then

$$
\infty=\sum_{\beta_{n} \geq a_{n}} a_{n}^{p} \leq \sum_{\beta_{n} \geq a_{n}} \beta_{n}^{p} \leq \sum_{n=1}^{\infty} \beta_{n}^{p}<\infty
$$

and we have a contradiction. Now, let $T\left(\left(\alpha_{n}\right)\right)=\sum_{n=1}^{\infty} \alpha_{n} x_{n}$ be the natural linear imbedding of $\ell_{1}$ into $X$. Then the formula $\tau(B)=\mu\left(T^{-1}(B)\right)$ for Borel subsets $B$ of $X$ defines a Borel probability measure on $X$. Let us show that $\tau(x-Q)=0$ for every translate $x-Q$ of the negative cone $-Q$. If $x=\sum_{n=1}^{\infty} \beta_{n} x_{n}$, then

$$
\tau(x-Q)=\mu\left\{\left(\alpha_{n}\right) \in \ell_{1}: \alpha_{n} \leq \beta_{n}\right\}=0,
$$

where the latter equality follows from the fact that if $\sum_{n=1}^{\infty} \beta_{n} x_{n} \in X$, then $\left(\beta_{n}\right) \in$ $\ell_{p}$ by (1). Hence, the negative cone $-Q$ is Haar null in $X$, and consequently so is $Q$.

We remark that the convexity assumption in the following lemma is essential; if $S$ is the unit sphere of a Banach space $X$ and $P$ a projection onto a subspace of $X$ then the relative interior of $P(S)$ is nonempty. The lemma holds also, by a result of Vašák $[\mathrm{V}]$ (see $[\mathrm{S}]$ for a different proof), for weakly countably determined Banach spaces. The proof of the lemma was suggested to us by C. Stegall.

Lemma 3. Let $X$ be a weakly compactly generated Banach space and $C$ a closed and convex subset of $X$ with empty interior. Then there exists a projection $P$ onto a separable subspace $Z$ of $X$ such that the interior of $\overline{P(C)}$ in $Z$ is empty.

Proof. Since $X$ is weakly compactly generated, by the theorem of Amir and Lindenstrauss [AL], there exists a sequence of projections $P_{n}: X \rightarrow X$ so that $P_{n}(X)$ is separable for all $n \in N, P_{m} P_{n}=P_{n} P_{m}=P_{\min \{m, n\}}$ for all $m, n \in N$, and $P(x)=\lim P_{n}(x)$ for $x \in X$ exists and is a projection onto $\overline{\cup P_{n}(X)}$. Moreover, one can construct these projections so that if $P_{n-1}$ is already constructed and $D_{n} \subset X$, $A_{n} \subset X^{*}$ are given separable sets then $P_{n}$ can be chosen so that $D_{n} \subset P_{n}(X)$ and $A_{n} \subset P_{n}^{*}\left(X^{*}\right)$ (see $\left.[\mathrm{AL}]\right)$. Let us construct by induction a sequence of such projections as follows. Let $P_{o}$ be any projection of $X$ onto a separable subspace. If $P_{n-1}$ was already constructed, choose a countable set $D_{n} \in X \backslash C$ so that $P_{n-1}(C) \subset \bar{D}_{n}$. This is possible, because the interior of $C$ is empty and $P_{n-1}(C)$ is separable. Since $C$ is closed and convex, each point of $D_{n}$ can be strictly separated from $C$ by some $x^{*} \in X^{*},\left\|x^{*}\right\|=1$. Therefore we can choose a countable set $A_{n}$ in the unit ball of $X^{*}$ so that for each $x \in D_{n}$ there exists some $x^{*} \in A_{n}$ and $\delta>0$ so that

$$
\left\langle x^{*}, x-z\right\rangle \geq \delta \text { for all } z \in C \text {. }
$$


Choose $P_{n}$ as above so that $D_{n} \subset P_{n}(X)$ and $A_{n} \subset P_{n}^{*}\left(X^{*}\right)$. From the continuity of the projections it follows, that

$$
\overline{P(C)} \subset \overline{\bigcup_{n=0}^{\infty} P_{n}(C)} \subset \overline{\bigcup_{n=1}^{\infty} D_{n}} .
$$

Also, $P(x)=x$ and $P^{*}\left(x^{*}\right)=x^{*}$ for all $x \in \bigcup D_{n}$ and $x^{*} \in \bigcup A_{n}$. Let $x \in \bigcup D_{n}$ be given; choose $\delta>0$ and $x^{*} \in \bigcup A_{n}$ so that (2) holds. Then,

$$
\begin{aligned}
\operatorname{dist}(x, \overline{P(C)}) & =\operatorname{dist}(x, P(C)) \geq \inf _{z \in C}\left\langle x^{*}, x-P(z)\right\rangle \\
& =\inf _{z \in C}\left\langle x^{*}, P(x)-P(z)\right\rangle=\inf _{z \in C}\left\langle P^{*}\left(x^{*}\right), x-z\right\rangle \\
& =\inf _{z \in C}\left\langle x^{*}, x-z\right\rangle \geq \delta>0 .
\end{aligned}
$$

Since $\overline{\cup D_{n}}$ contains $P(C)$ and each point of $\bigcup D_{n}$ has positive distance to $\overline{P(C)}$, the interior of $\overline{P(C)}$ is empty.

The proof of the following theorem shows that if $X$ is a reflexive Banach space with the property that the positive cone of each quotient of $X$ with Schauder basis is Haar null then for every closed and convex $C \subset X$ with empty interior there exists a Radon probability measure $\mu$ on $X$ so that $\mu(C+x)=0$ for all $x \in X$. Superreflexive spaces have this property, since basic sequences in superreflexive spaces satisfy estimates similar to (1) in Lemma 2. Namely, a Banach space is superreflexive if and only if $0<2 k<\varepsilon \leq 1<K$; then there exist $p, q>1$ such that for any normalized basic sequence $\left\{x_{n}\right\}$ in $X$ with characteristic not less then $\varepsilon$ we have

$$
k\left(\sum_{i=1}^{n}\left|\beta_{i}\right|^{p}\right)^{\frac{1}{p}} \leq\left\|\sum_{i=1}^{n} \beta_{i} x_{i}\right\| \leq K\left(\sum_{i=1}^{n}\left|\beta_{i}\right|^{q}\right)^{\frac{1}{q}}
$$

for any real numbers $\beta_{1}, \ldots, \beta_{n}$ (see $[\mathrm{GG}]$, or $[\mathrm{J}]$ ).

Theorem 4. Let $X$ be a superreflexive Banach space and $C \subset X$ a closed and convex set with empty interior. Then there exists a Radon probability measure $\mu$ on $X$ so that $\mu(C+x)=0$ for all $x \in X$. In particular, in separable superreflexive Banach spaces, closed and convex sets with empty interior are Haar null.

Proof. As in the previous lemma, let $P$ be a projection of $X$ onto a separable subspace $Z$ of $X$ such that the interior of $\overline{P(C)}$ in $Z$ is empty. We will show that $\overline{P(C)}$ is Haar null in $Z$. Then, if $\mu$ is a Borel probability measure on $Z$ for which $\mu(\overline{P(C)}+z)=0$ whenever $z \in Z$ we can extend $\mu$ on Borel subsets of $X$ by the formula $\mu(A)=\mu(A \cap Z)$. Then $\mu$ has the required properties since $(C+x) \cap Z \subset P(C+x) \subset \overline{P(C)}+P(x)$ for each $x \in X$. Since a countable union of Haar null sets is Haar null, we can suppose that $D=\overline{P(C)}$ is bounded. If $D$ is not spanning in $Z$, there exists $z \in Z$ so that the intersection of any translate of the line segment $[0, z]$ and $D$ contains at most one point. Consequently, any translate of $D$ is a null set for the Lebesgue measure sitting on $[0, z]$. Suppose that $D$ is spanning. By Lemma 1 there exists a quotient space $Y=T(Z)$ of $Z$ with a basis $\left\{y_{n}\right\}$ and $v \in Z$ so that $T(D+v)$ is contained in the positive cone $Q$ of $Y$, where $T$ is the quotient mapping. By considering $\left\{y_{n} /\left\|y_{n}\right\|\right\}$ instead of $\left\{y_{n}\right\}$, we can suppose that the basis of $Y$ is normalized. Since $X$ is superreflexive, $Y$ is also superreflexive, and consequently the basis admits an estimate of type (1). Hence the positive 
cone $Q$ of $Y$ is by Lemma 2 Haar null; denote by $\nu$ a probability measure on $Y$, for which $\nu(y+Q)=0$ for all $y \in Y$. Since $T$ is a surjective continuous linear operator, the inverse of $T$ admits, by a theorem of Bartle and Graves (see [BP]), a continuous selection $f$. Consequently, the formula $\tau(A)=\nu\left(f^{-1}(A)\right)$ for Borel subsets $A$ of $Z$ defines a probability measure on $Z$. Let $z \in Z$ be given. Then $f^{-1}(D+z) \subset T(D+v)+T(z-v) \subset Q+T(z-v)$, and consequently

$$
\begin{aligned}
\tau(D+z) & =\nu\left(f^{-1}(D+z)\right) \leq \nu(T(D+v)+T(z-v)) \\
& \leq \nu(Q+T(z-v))=0 .
\end{aligned}
$$

Hence $\tau(D+z)=0$ for each $z \in Z$, and $D$ is a Haar null set.

We wish to thank L. Zajíček for directing our attention to this problem, and C. Stegall for help with Lemma 3.

\section{REFERENCES}

[A] N. Aronszajn, Differentiability of Lipschitzian mappings between Banach spaces, Studia Math. LVII (1976), 147-190. MR 54:13562

[AL] D. Amir and J. Lindenstrauss, The structure of weakly compact sets in Banach spaces, Ann. of Math. 88 (1968), 35-46. MR 37:4562

[BP] C. Bessaga and A. Pelczynski, Selected topics in infinite-dimensional topology, PWN, Warszawa 1975. MR 57:17657

[BN] J.M. Borwein and D. Noll, Second order differentiability of convex functions in Banach spaces, Trans. A.M.S. 342 (1994), 43-81. MR 94e:46076

[C] J.P.R. Christensen, Topology and Borel structure, North-Holland, Amsterdam 1974. MR 50:1221

[GG] V.I. Gurarii and N.I. Gurarri, On basis in uniformly convex and uniformly smooth spaces, Izv. Akad. Nauk SSSR 35 (1971), 210-215. MR 44:780

[J] R.C. James, Super-reflexive spaces with bases, Pacific J. of Math., Vol. 41, 2 (1972), 409-419 MR 46:7866

[LT] J. Lindenstarauss and L. Tzafriri, Classical Banach spaces I., Springer-Verlag, Berlin 1977. MR 58:17766

[MS] E. Matoušková and C. Stegall, A characterization of reflexive Banach spaces, to appear in Proc. A.M.S. MR 96g: 46005

[MZ] E. Matoušková and L. Zajíček, Second order differentiability of integral functionals, to appear in Czechoslov. Math. Journ.

$[\mathrm{PT}]$ D. Preiss and J. Tišer, Two unexpected examples concerning differentiability of Lipschitz functions on Banach spaces, to appear in Proceedings of GAFA seminar, Tel Aviv.

[S] C. Stegall, A proof of the theorem of Amir and Lindenstrauss, Israel Jour. of Math., 68 (1989), 185-192. MR 91b:46017

[V] L. Vašák, On one generalization of weakly compactly generated Banach spaces, Studia Math. 70 (1981), 11-19. MR 83h:46028

Department of Mathematical Analysis, Charles University, Sokolovská 83,18600 Prague, Czech Republic

Institut für Mathematik, Johannes Kepler Universität, Altenbergerstrasse, A-4040 LinZ, Austria

E-mail address: eva@caddo.bayou.uni-linz.ac.at 